\title{
Prevalence and risk factors for nocturia in middle-aged and elderly people from public health centers in Taiwan
}

\author{
Mei-Huang Huang, Aih-Fung Chiu, Chung-Cheng Wang, Hann-Chorng Kuo \\ Department of Nursing, Meiho University (MHH), Pingtung; Institute of Medical Science, Tzu Chi \\ University (AFC), Pingiung ; Department of Urology, En Chu Kong Hospital and Department of Biomedical \\ Engineering, Chung Yuan Christian University (CCW), New Taipei City and Department of Urology, \\ Buddhist Tzu Chi General Hospital and Tzu Chi University (HCK), Hualien, Taiwan
}

\section{ABSTRACT}

Purpose: To assess the occurrence and the associated risk factors for nocturia among middle-aged and elderly people from public health centers in southern Taiwan.

Materials and Methods: Data were part of our previous cross-sectional study which used a self-administered questionnaire for the assessment of lower urinary tract symptoms. A total of 1011 responders who were at least 40 years of age were enrolled from any of four local public health centers for any reason in Pingtung County, Taiwan. Nocturia, as a dependent variable, was defined as two or more episodes per night. Covariables included age, gender, and chronic illnesses (obesity, hypertension, diabetes, cardiovascular disease, and stroke). Multivariate logistic regression was applied to determine the risk factors associated with nocturia. A p-value of less than 0.05 was considered statistically significant.

Results: About 38.1\% (385/1011) of the participants reported having nocturia $\geq 2$ episodes/night, and the occurrence of nocturia increased with advanced age. More than half (51.2\%, 197/385) participants with nocturia perceived at least "a bit of a problem" on the sleep quality. The multivariate logistical regression showed that the independent risk factors for nocturia were age (OR:1.06, CI:1.05-1.08), hypertension (OR:1.58, CI:1.16-2.16) and diabetes (OR:1.59, CI:1.03-2.45) and obesity (OR:1.47, CI:1.02-2.10), while a borderline effect on nocturia was produced by cardiovascular disease (OR:1.66, CI: 0.98-2.79) and stroke (OR:2.75, CI:0.88-8.64).

Conclusions: Several chronic illnesses coexisted with nocturia. Health care providers need to be aware of an increased risk of nocturia among people with certain chronic illnesses, and provide appropriate health care.

\section{ARTICLE INFO}

\section{Key words:}

Nocturia, Comorbidity,

Hypertension, Diabetes

Mellitus, Obesity

Int Braz J Urol. 2012; 38: 818-24

Submitted for publication:

March 06, 2012

Accepted after revision: July 09, 2012

\section{INTRODUCTION}

In 2002, the International Continence Society (ICS) proposed the first standardization of nocturia terminology. Since then, nocturia has begun to be recognized as an entire clinical concern, instead of just a lower urinary tract symptom. Based on the ICS de- finition, nocturia is defined as an individual needing to wake from sleep to void (1). Nocturia can cause sleep disturbances, lead to poor physical and mental health $(2,3)$, and even increase the mortality risk (4).

Nocturia is age-related and both genders have a similar prevalence (5-7). In previous epidemiologic surveys, the prevalence of nocturia $(\geq$ 
2 episodes per night) showed great variation, from $10 \%$ (5) to $30 \%(6,7)$, depending upon the study population and methodology. Despite the variations, the reports revealed that nocturia is a non-specific, but common urinary symptom.

People who suffer from nocturia are usually older and may also have other chronic illnesses. Clinical observations have indicated that some chronic illnesses are associated with nocturia. The Tikkinen et al. study reported that being overweight or obese, having coronary artery disease or diabetes in women and obesity in men, were significantly associated with nocturia in Finland (8). The Gourova et al. study revealed that cardiovascular disease, hypertension, diabetes mellitus/insipidus, and cerebrovascular disease were independent risk factors for nocturia in men aged 55-75 years in the Netherlands (9). The BACH study showed that nocturia was significantly associated with an increasing body mass index, type II diabetes, cardiac disease, and diuretics use (7). However, in a community-dwelling elderly population study conducted in Sweden, there was no correlation between nocturia and a known and treated hypertension, angina pectoris, congestive heart failure or diabetes mellitus (10). The similar finding was also noted by a Dutch study that nocturia was not associated with cardiovascular symptoms, hypertension or diabetes mellitus in men (11). Furthermore, base on frequency-volume chart data, Doorn et al. also found different results that nocturia did not necessarily related to increased mortality risk (12). Thus, some discrepancy exists among previous reports.

From the viewpoints of public health and disease prevention, it is important to identify the risk factors of nocturia so that effective prevention or treatments can be given when needed. In addition, to our knowledge, most published literatures were conducted in the western world (7-11). The data from Asia are relatively limited. Thus, we investigated the prevalence and risk factors of nocturia in middle-aged and elderly people in Taiwan.

\section{MATERIALS AND METHODS}

\section{Design and sample}

The data were part of our previous cross-sectional study which used a self-administered questionnaire to assess lower urinary tract symptoms (13). For this study, based on a convenient sampling, adults who were 40 or more years of age and had visited any of the local four public health centers for a physical checkup in Pingtung County, Taiwan, were enrolled from March to July of 2010. The inclusion criteria were voluntary participation and being free of severe disabilities related to hearing, seeing, speaking, and walking. People who complained of active urinary tract infections or micturition pain were excluded.

Of the 1190 subjects who were contacted, 135 subjects who did not complete the survey, and 44 subjects who complained of active urinary tract infection or micturition pain were excluded. As a result, 1011 participants (539 women and 472 men) were included in this analysis. The sample was designed to yield results within $\pm 3 \%$ error with $95 \%$ confidence level.

\section{Measurement}

As an outcome measure, the measure of nocturia was based on the nocturia item in the International Prostate Symptom Score. Since two episodes of nocturia constitute meaningful nocturia affecting well-being and perceived health (14), we defined nocturia as two or more episodes per night. Covariables included age, gender, and chronic illnesses. Chronic illnesses were assessed by asking the participants whether their doctors had told them about the following diseases: hypertension, diabetes, heart disease (congestive heart failure, myocardial infarction, angina, coronary artery bypass, or angioplasty), and stroke. The body weight and height were measured after interview and obesity was recorded if participants had a body mass index of $>27 \mathrm{~kg} / \mathrm{m}^{2}$. In addition, each participant was asked "How much of a problem does nocturia pose to your quality of sleep?" Their responses were (based on "not at all", "a bit of a problem", "quite a problem", and "a serious problem") were also documented.

The study was approved by the Institutional Review Board of a local university in Pingtung, Taiwan, and written informed consent was obtained from all participants. During the interviews, if any participant felt uncomfortable and wanted to discontinue the interview, he or she had the right to do so. 


\section{Data analysis}

Continuous variables are presented as the mean \pm standard deviation (SD), while categorized variables are presented as number \pm percentage $(\%)$ and were compared using the chi-squared test. We used multivariable logistic regression analysis controlling age, gender, and all chronic illnesses (obesity, hypertension, diabetes, heart disease, and stroke) to explore the significance of the factors associated with nocturia of $\geq 2$ episodes/night, and calculated the odds ratio (OR) and 95\% confidence interval (95\% CI). The Hosmer-Lemeshow $\chi^{2}$ test was used to test the logistic regression model fit. Statistical analyses were performed using SPSS for Windows (version 17.0; SPSS Inc, Chicago, IL, USA). A p-value of less than 0.05 was considered statistically significant.

\section{RESULTS}

The mean age of the 1011 participants (539 women and 472 men) was $61.5 \pm 12.0$ (medium 61, range 40-93). The prevalence of nocturia ( $\geq 2$ episodes/night) according to the distribution of age and gender is shown in Table-1. Overall, 38.1\% $(n=385)$ of the individuals answered that they arose for urination during the night at least twice. Excepting the sixth decade ( $47.4 \%$ vs. $33.6 \%, \chi^{2}=4.76, p=0.029$ ), both genders had similar occurrence of nocturia on other age groups. Facing substantial participants with nocturia, about half $(51.2 \%, 197 / 385)$ of them perceived at least "a bit of a problem" with their sleep being impaired by nocturia (Table-2).

Table-3 reveals the prevalence of nocturia ( $\geq 2$ episodes/night) in the potential factors and the multivariate logistic analysis for the risk factors of nocturia. The highest percentage of nocturia occurred in participants with stroke (81.0\%), followed by diabetes (60.7\%), and cardiovascular disease $(60.1 \%)$. The multivariate logistic regression showed that nocturia was independently associated with increased age (OR 1.06, 95 \% CI: 1.05-1.08), hypertension (OR 1.58, 95 \% CI: 1.16-2.16), diabetes (OR 1.59, 95 \% CI: 1.03-2.45), and obesity (OR 1.47, 95 $\%$ CI 1.02-2.10), while cardiovascular disease (OR 1.66, 95 \% CI: 0.98-2.79) and stroke (OR 2.75, $95 \%$ CI: 0.88-8.64) had borderline effects on nocturia.

\section{DISCUSSION}

Our findings revealed that in the studied sample with a mean age of 60 years, approximately $38 \%$ of participantants had nocturia twice or more per night, and half $(51.2 \%, 197 / 385)$ of them perceived at least "a bit of a problem" with their sleeping quality. Facing the fact that the percentage of the population who are reaching middle or old age is rapidly rising in Taiwan, health care providers should be more cognizant of nocturia.

In addition to age, which had a strong association with nocturia in this study, several

Table 1 - Prevalence of nocturia ( $\geq 2$ episodes/night) by age and gender.

\begin{tabular}{lccccccc}
\hline \multirow{2}{*}{ Age } & \multicolumn{2}{c}{ Both genders } & \multicolumn{2}{c}{ Women } & \multicolumn{2}{c}{ Men } & \multicolumn{2}{c}{ p-value } \\
\hline & $\mathrm{N}$ & $(\%)$ & $\mathrm{N}$ & $(\%)$ & $\mathrm{N}$ & $(\%)$ & \\
\cline { 2 - 6 } $40-49$ & $28 / 197$ & $(14.2)$ & $18 / 119$ & $(15.1)$ & $10 / 78$ & $(12.8)$ & 0.650 \\
$50-59$ & $74 / 273$ & $(27.1)$ & $42 / 147$ & $(28.6)$ & $32 / 126$ & $(25.4)$ & 0.556 \\
$60-69$ & $97 / 242$ & $(40.1)$ & $43 / 128$ & $(33.6)$ & $54 / 114$ & $(47.4)$ & $0.029^{*}$ \\
$\geq 70$ & $186 / 299$ & $(62.2)$ & $90 / 145$ & $(62.1)$ & $96 / 154$ & $(62.3)$ & 0.962 \\
\hline Total & $385 / 1011$ & $(38.1)$ & $193 / 539$ & $(35.8)$ & $192 / 472$ & $(40.7)$ & 0.112 \\
\hline
\end{tabular}

Chi-squared tests were used.

*indicated $p<0.05$. 
Table 2 - The impact on sleeping between participants with and without nocturia ( $\geq 2$ episodes/night).

\begin{tabular}{|c|c|c|c|c|c|c|}
\hline & \multirow[t]{2}{*}{ Total } & \multicolumn{2}{|c|}{$\begin{array}{c}\text { Non-nocturia } \\
\text { (<2 episodes/night) }\end{array}$} & \multicolumn{2}{|c|}{$\begin{array}{c}\text { Nocturia } \\
\text { ( } \geq 2 \text { episodes/night) }\end{array}$} & \multirow[t]{2}{*}{$p$-value } \\
\hline & & & & & & \\
\hline Sleeping quality & & N & $(\%)$ & N & $(\%)$ & $0.000^{*}$ \\
\hline Not a problem & 702 & 514 & $(82.1)$ & 188 & $(48.8)$ & \\
\hline A bit of a problem & 231 & 100 & $(16.0)$ & 131 & $(34.0)$ & \\
\hline Quite a problem & 58 & 11 & (1.8) & 47 & $(12.2)$ & \\
\hline A serious problem & 20 & 1 & $(0.2)$ & 19 & $(4.9)$ & \\
\hline
\end{tabular}

Chi-squared test was used.

*indicated $p<0.05$.

Table 3 - Prevalence of nocturia ( $\geq 2$ episodes/night) in potential factors and the analysis for the risk factors of nocturia.

\begin{tabular}{|c|c|c|c|c|c|}
\hline & \multicolumn{2}{|c|}{ With nocturia (\%) } & $\mathrm{OR}$ & $(95 \% \mathrm{Cl})$ & $p$-value \\
\hline Age & & & 1.06 & $(1.05-1.08)$ & $0.000^{*}$ \\
\hline \multicolumn{6}{|l|}{ Gender (reference: female) } \\
\hline Women $(n=539)$ & 193 & $(35.8)$ & 1 & & \\
\hline Men $(n=472)$ & 192 & $(40.7)$ & 1.13 & $(0.85-1.49)$ & 0.399 \\
\hline Hypertension $(n=304)$ & 167 & $(54.9)$ & 1.58 & $(1.16-2.16)$ & $0.004^{*}$ \\
\hline Diabetes $(n=117)$ & 71 & $(60.7)$ & 1.59 & $(1.03-2.45)$ & $0.035^{\star}$ \\
\hline Cardiovascular disease $\dagger(n=75)$ & 45 & $(60.0)$ & 1.66 & $(0.98-2.79)$ & 0.059 \\
\hline Stroke history $(n=21)$ & 17 & $(81.0)$ & 2.75 & $(0.88-8.64)$ & 0.083 \\
\hline Obesity $(n=180)$ & 86 & $(47.8)$ & 1.47 & $(1.02-2.10)$ & $0.038^{\star}$ \\
\hline
\end{tabular}

Multivariate logistical regression was used and adjusted for all variables mentioned above.

† Includes congestive heart failure, myocardial infarction, angina, coronary artery bypass, or angioplasty.

* Statistically significant.

chronic illnesses were significantly associated with nocturia ( $\geq 2$ episodes/night), which implied that the nocturia may be multi-factorial in origin. Hypertension was significantly associated with nocturia, similar to the findings of some previous studies $(6,9,15)$, but not others $(10,11)$. Based on nocturnal polyuria, an important pathogenetic factor characterized by natriureses, McKeigue and Reynard suggested that hypertension caused by increased urolitidin secretion can depress the circadian rhythm of sodium secretion and lead to nocturnal polyuria (16). However, the mechanisms between them are not completely clear.

Diabetes mellitus was another independent predictor for nocturia. The result is congruent with numerous previous studies $(6-9,15,17,18)$, 
but a few studies have disagreed $(10,11)$. The osmotic diuresis and global polyuria induced by hyperglycemia in diabetes patients has been demonstrated as the major cause of urinary storage symptoms (19). Thus, it is logical to hypothesize that the association between the effects of hyperglycemia may, at least in part, affect the occurrence of nocturia. In addition, the lack of arginine vasopressin (AVP) secretion and renal AVP resistance induced by diabetes may play a crucial role (20,21).

People with obesity (BMI of $>27 \mathrm{~kg} / \mathrm{m}^{2}$ ) had higher odds for suffering from nocturia, as compared to those without obesity, which was consistent with numerous epidemiological studies $(5,7,8,15,17)$. The exact mechanism by which obesity causes nocturia is not known. There is a relationship between sleep apnea and nocturia (2224), and both are associated with obesity. Obesity can induce both sleep apnea and hypoxia, as well as possibly increasing atrial natriuretic peptide (ANP). Thus, the mechanism of natriuresis among obeses people with sleep apnea and hypoxia should not be overlooked (25) and may account for these associations.

Heart disease had borderline significance with nocturia, which may be resulted from the relatively small number of people with heart disease $(n=75)$. Some earlier epidemiologic studies reported significant associations of heart disease with nocturia (7-9), while others did not $(6,10,11,17)$. A recent study conducted by Chiu et al. demonstrated that patients with chronic heart failure suffer from three or more episodes of nocturia as compared to the controls (26). Possible reasons such as postural diuresis at night, nocturnal polyuria as a result of an increase in the atrial natriuretic peptide (ANP), or wrong timing of diuretics therapy (particularly loop diuretics) (25) may account for the association.

Only 25 subjects reported to have had stroke and there was also a borderline effects of stroke $(n=25)$ with nocturia. Two studies rejected the association of nocturia with stroke $(6,17)$. However, a previous study conducted by Asplund demonstrated that stroke was significantly associated with nocturia of $\geq 3$ voids (18). Also, Brittain et al. reported that nocturia was the most common urinary symptom, and that stroke survivors had a higher prevalence of nocturia than the nonstroke population (49\% vs. 19\%, respectively) (27). The associations of stroke with nocturia may be partially explained by the lack of the normal nocturnal rise of plasma AVP in the post-stroke patient (28) and the uninhibited detrusor contractions secondary to the neurogenic defect. However, the actual mechanisms between them are not completely clear.

The causes for nocturia were previously classified as diurnal polyuria, nocturnal polyuria, low functional bladder capacity, or a combination of these, and the plasma AVP and ANP may be crucial humoral factors $(21,29)$. In our study, nocturia was significantly associated with several illnesses, and some plausible mechanisms or hypotheses are proposed to explain these relationships. Besides, psychogenic nocturia, sleep apnea, caffeine intake, alcohol intake, bladder outlet obstruction and detrusor overactivity have been considered as possible etologies for nocturia. Additional studies regarding these aspects in the future may also be needed.

There are some limitations in the present study. First, this analysis was based on participants' reports rather than voiding diary or the measure of functional changes of the bladder. Recently in the Krimpen study, Doorn et al. also showed that the discrepancy during assessing nocturia was significant between questionaire and voiding diary (30). Thus, the recall bias must be considered. Second, all participants were recruited from public health centers, not from hospitals. Most participants could not provide the exact drug names. Thus, the effect of medications (eg, diuretics) on nocturia could not be understood in our study. Third, our study enrolled participants from public health centers, where the patients studied tended to be either elderly or patients with chronic illnesses. Consequently, the prevalence of 38.1\% for nocturia may be higher than the epidemiologic rate of nocturia (5-7). We focused here on the association between nocturia and some specific chronic illnesses, rather than the truly epidemiologic rate. Overall, despite these limitations, the findings of this study suggest relevance to clinical practice and directions for further research. 


\section{CONCLUSIONS}

In our study, nocturia was significantly associated with several illnesses, and some plausible mechanisms or hypotheses are proposed to explain these relationships. When treating people with nocturia, health care providers need to be aware of the possible non-urogenital reasons for the nocturia, and provide appropriate health care.

\section{CONFLICT OF INTEREST}

None declared.

\section{REFERENCES}

1. Van Kerrebroeck P, Abrams P, Chaikin D, Donovan J, Fonda $D$, Jackson $S$, et al.: The standardization of terminology in nocturia: report from the standardization subcommittee of the International Continence Society. BJU Int. 2002; 90(Suppl 3): 11-5.

2. Schneider T, Stanley N: Impact of nocturia on sleep and energy. Eur Urol Suppl. 2007; 6: 585-93.

3. Coyne KS, Zhou Z, Bhattacharyya SK, Thompson CL, Dhawan $\mathrm{R}$, Versi $\mathrm{E}$ : The prevalence of nocturia and its effect on health-related quality of life and sleep in a community sample in the USA. BJU Int. 2003; 92: 948-54.

4. Kupelian V, Fitzgerald MP, Kaplan SA, Norgaard JP, Chiu GR, Rosen RC: Association of nocturia and mortality: results from the Third National Health and Nutrition Examination Survey. J Urol. 2011; 185: 571-7.

5. Schatzl G, Temml C, Schmidbauer J, Dolezal B, Haidinger $G$, Madersbacher S: Cross-sectional study of nocturia in both sexes: analysis of a voluntary health screening project. Urology. 2000; 56: 71-5.

6. Yoshimura K, Terada N, Matsui Y, Terai A, Kinukawa N, Arai $Y$ : Prevalence of and risk factors for nocturia: Analysis of a health screening program. Int J Urol. 2004; 11: 282-7.

7. Fitzgerald MP, Litman HJ, Link CL, McKinlay JB; BACH Survey Investigators: The association of nocturia with cardiac disease, diabetes, body mass index, age and diuretic use: results from the BACH survey. J Urol. 2007; 177: 1385-9.

8. Tikkinen KA, Auvinen A, Johnson TM 2nd, Weiss JP, Keränen T, Tiitinen A, et al.: A systematic evaluation of factors associated with nocturia--the population-based FINNO study. Am J Epidemiol. 2009 1; 170: 361-8.

9. Gourova LW, van de Beek C, Spigt MG, Nieman FH, van Kerrebroeck PE: Predictive factors for nocturia in elderly men: a cross-sectional study in 21 general practices. BJU Int. 2006; 97: 528-32.
10. Rembratt A, Norgaard JP, Andersson KE: Nocturia and associated morbidity in a community-dwelling elderly population. BJU Int. 2003; 92: 726-30.

11. Blanker MH, Bernsen RM, Ruud Bosch JL, Thomas S, Groeneveld FP, Prins A, Bohnen AM: Normal values and determinants of circadian urine production in older men: a populationbased study. J Urol. 2002; 168(4 Pt 1): 1453-7.

12. van Doorn B, Kok ET, Blanker MH, Westers P, Bosch JL: Mortality in older men with nocturia. A 15-year followup of the Krimpen study. J Urol. 2012; 187: 1727-31.

13. Chiu AF, Huang MH, Wang CC, Kuo HC: Higher glycosylated hemoglobin levels increase the risk of overactive bladder syndrome in patients with type 2 diabetes mellitus. Int $\mathrm{J}$ Urol. 2012; 19: 995-1001.

14. Tikkinen KA, Johnson TM 2nd, Tammela TL, Sintonen H, Haukka J, Huhtala $\mathrm{H}$, et al.: Nocturia frequency, bother, and quality of life: how often is too often? A population-based study in Finland. Eur Urol. 2010; 57: 488-96.

15. Hsieh CH, Chen HY, Hsu CS, Chang ST, Chiang CD: Risk factors for nocturia in Taiwanese women aged 20-59 years. Taiwan J Obstet Gynecol. 2007; 46: 166-70.

16. McKeigue PM, Reynard JM: Relation of nocturnal polyuria of the elderly to essential hypertension. Lancet. 2000; 355: 486-8.

17. Bing MH, Moller LA, Jennum P, Mortensen S, Lose G: Nocturia and associated morbidity in a Danish population of men and women aged 60-80 years. BJU Int. 2008; 102: 808-14; discussion 814-5.

18. Asplund R: Nocturia in relation to sleep, somatic diseases and medical treatment in the elderly. BJU Int. 2002; 90: 533-6.

19. Daneshgari F, Liu G, Birder L, Hanna-Mitchell AT, Chacko S: Diabetic bladder dysfunction: current translational knowledge. J Urol. 2009; 182(6 Suppl): S18-26.

20. Bankir L, Bardoux P, Ahloulay M: Vasopressin and diabetes mellitus. Nephron. 2001; 87: 8-18.

21. Schneider T, de la Rosette JJ, Michel MC: Nocturia: a nonspecific but important symptom of urological disease. Int $\mathrm{J}$ Urol. 2009; 16: 249-56.

22. Fitzgerald MP, Mulligan M, Parthasarathy S: Nocturic frequency is related to severity of obstructive sleep apnea, improves with continuous positive airways treatment. Am J Obstet Gynecol. 2006; 194: 1399-403.

23. Hajduk IA, Strollo PJ Jr, Jasani RR, Atwood CW Jr, Houck $\mathrm{PR}$, Sanders MH: Prevalence and predictors of nocturia in obstructive sleep apnea-hypopnea syndrome--a retrospective study. Sleep. 2003 1; 26: 61-4.

24. Umlauf MG, Chasens ER, Greevy RA, Arnold J, Burgio KL, Pillion DJ: Obstructive sleep apnea, nocturia and polyuria in older adults. Sleep. 2004; 27: 139-44.

25. Aydur E, Dmochowski R: Medical Conditions Associated with Nocturia. In: Weiss JPP, Blaivas JGG, Van Kerrebroeck PEVEV, Wein AJJ, (ed.), Nocturia. Springer New York. 2012; pp. 11-36. 
26. Chiu AF, Liao $\mathrm{CH}$, Wang CC, Wang JH, Tsai CH, Kuo HC: High classification of chronic heart failure increases risk of overactive bladder syndrome and lower urinary tract symptoms. Urology. 2012; 79: 260-5.

27. Brittain KR, Perry SI, Peet SM, Shaw C, Dallosso H, Assassa RP, et al.: Prevalence and impact of urinary symptoms among community-dwelling stroke survivors. Stroke. 2000; 31: 886-91.
28. Sakakibara R, Uchiyama T, Liu Z, Yamamoto T, Ito T, Yamanishi $T$, et al.: Nocturnal polyuria with abnormal circadian rhythm of plasma arginine vasopressin in post-stroke patients. Intern Med. 2005; 44: 281-4.

29. Weiss JP, Blaivas JG: Nocturia. J Urol. 2000; 163: 5-12.

30. van Doorn B, Blanker MH, Kok ET, Westers P, Bosch JL: Once nocturia, always nocturia? Natural history of nocturia in older men based on frequency-volume charts: the Krimpen study. J Urol. 2011; 186: 1956-61.

\section{Correspondence address:}

Dr. Hann-Chorng Kuo Department of Urology

Buddhist Tzu Chi General Hospital, 707, Section 3 Chuang Yang Road, Hualien, Taiwan Fax: + 03856 1825-2113

E-mail: hck@tzuchi.com.tw 